\author{
M.J. Sablik \\ Applied Magnetics and Physical Modeling, 78240 San Antonio, TX, U.S.A \\ msablik55@outlook.com
}

\title{
PREDICTION OF MAGNETIC PROPERTIES OF A PLASTICALLY DEFORMED STEEL AND ONE WAY TO MEASURE ITS PLASTIC DEFORMATION
}

\begin{abstract}
In this paper, we use a phenomenological model based on the Jiles-Atherton-Sablik model of stress affecting the magnetic hysteresis of magnetic materials as modified when stress goes past the yield stress We use this to show that (1) the model produces sharp shearing of hysteresis curves, as seen experimentally and that (2) it also produces a step in the hysteresis loss at small residual plastic strain. We also find that the step in the hysteresis loss can be fitted to a power law, and find that the power law can be fitted by the power $m=0.270$, different from the mechanical Ludwik Law exponent, and reasonably close to the experimental 0.333 and 0.202 . We will also suggest a method of measuring how plastically deformed the material is by suggesting how the dislocation density can be measured.
\end{abstract}

Keywords: Steel plastic deformation, magnetic hysteresis modeling, nondestructive evaluation

\section{INTRODUCTION}

This paper deals with evaluation of the microstructure state of steels using an electromagnetic approach. We approach this problem by using modeling to understand the phenomena. By understanding the effect of changed microstructure on the magnetic properties of materials, we can use those properties to quantify the amount of plastic damage in a material. One of the things shown by this paper is how to quantify the plastic damage using magnetic properties.

In this paper, we use a model based on the Jiles-Atherton-Sablik model of stress affecting the magnetic hysteresis of magnetic materials,[1] as modified when stress goes past the yield stress [2]. We use this to show that (1) the model produces sharp shearing of hysteresis curves, as seen experimentally[2,3] and that (2) it also produces a sharp increase in the hysteresis loss at small 
residual plastic strain. We actually find that the hysteresis loss can be fitted to a power law, fitted by the power $m=0.270$, different from the mechanical Ludwik Law exponent, and reasonably close to the experimental 0.333 and 0.292 [4].

We will also suggest a method of measuring how plastically deformed the material is by suggesting how the dislocation density can be measured.

\section{THE MODEL}

The model has been used and modified by many authors over the years [5-10]. Our approach is to use the theory that was used in [4].

To model the plastic deformation, we start with total magnetization $\mathrm{M}$ as the sum of a reversible $\left(M_{r e v}\right)$ and an irreversible $\left(M_{i r r}\right)$ component. These components are given by

$$
\begin{aligned}
& M_{r e v}=c\left(M_{a}-M_{i r r}\right), \\
& M_{i r r}=M_{a}-\left(k \delta / \mu_{0}\right) d M_{i r r} / d H_{e} .
\end{aligned}
$$

Here, $M_{a}$ is the anhysteretic magnetization, given as

$$
M_{a}=M_{s} L\left(H_{e} / a\right),
$$

where $L(x)=\operatorname{coth} x-1 / x$ is the so-called Langevin function, and $H_{e}$ is the effective magnetic field in the material, i.e. [1]

$$
H_{e}=H+\alpha M_{a}+\left(3 \sigma / 2 \mu_{0}\right) d \lambda\left(M_{a}\right) / d M_{a} .
$$

In this, Ms, $\mathrm{c}, \mathrm{a}, \mathrm{k}$ and $\alpha$ are all parameters of the material. The parameter $\delta$ is +1 or -1 , depending on whether $\mathrm{H}$ is increasing or decreasing. In the last term in (4), $\sigma$ is the elastic stress acting, $\mu_{0}$ is permeability of free space, and $\lambda\left(M_{a}\right)$ is the magnetostriction in the anhysteretic equilibrium state. The last factor in (4) represents the derivative of the anhysteretic magnetostriction with respect to the magnetization in the anhysteretic state. The magnification $\mathrm{M}$ which is the sum of a reversible and irreversible component can be rewritten as a differential equation for $d M_{i r r} / d H$. It is seen that the equations have to be solved self-consistently in numerical fashion.

Since $k$ mathematically controls the amount of hysteresis, it is proportional to the coercivity and has the same dependences as the coercivity. Thus, we write, as formulated previously, [5]

$$
k=\left[G_{1}+G_{2} / d\right] \zeta_{d}^{1 / 2} k_{0}
$$

For $\mathrm{d}=\mathrm{d}^{*}=20 \mu \mathrm{m}$ and $\zeta_{d}=\zeta_{d}^{*}=1 \times 10^{12} / \mathrm{m}^{2}$, we choose $G_{1}$ and $G_{2}$ so that 


$$
\left[G_{1}+G_{2} / d^{*}\right]\left(\zeta_{d}^{*}\right)^{1 / 2}=1
$$

A choice that satisfies this is $G_{2}=10 \times 10^{-12} \mathrm{~m}^{2}$ and $G_{1}=0.5 \times 10^{-6} \mathrm{~m}$. Other choices for $G_{2}$ and $G$ also satisfy (6). The various choices correlate with the amount of spread in the hysteresis behavior due to variation of grain size $\mathrm{d}$ and dislocation density $\zeta_{d}$.

Note that when $\mathrm{d}$ and $\zeta_{d}$ satisfy (6) (i.e. when $d=d^{*}$ and $\zeta_{d}=\zeta_{d}{ }^{*}$ ), then $k=k_{o}$. The choices for $d^{*}$ and $\zeta_{d}^{*}$ represent a typical grain size and dislocation density that have been found in typical specimens of undeformed steel.

Scaling constant a is proportional to domain density in the demagnetized state, which is determined by pinning site density, which is in turn proportional to pinning constant $\mathrm{k}$. Thus, a has the same dependence on $\mathrm{d}$ and $\zeta_{d}$ that $k$ has, and hence

$$
a=\left\{\left[G_{3}+G_{4} / d\right] \zeta_{d}^{1 / 2}\right\} a_{0} .
$$

We have actually showed mathematically in an isotropic nontextured material that $a$ is directly proportional to $H_{c}$ and hence $k$. Thus, if we define $G_{3}$ and $G_{4}$ in the same way as $G_{1}$ and $G_{2}$, using (7) for $d=d$ and $\zeta_{d}=\zeta_{d}$, then we can set $G_{3}=G_{1}$ and $G_{4}=G_{2}$ and determine $a$.

In the relaxed state after deformation, where there is no applied stress, there is still a residual plastic strain $\varepsilon_{r}$. This means that there is an additional contribution to the magnetostrictive term in (4), which really devolves from the plastically modified magnetostrain energy.

We represent this additional contribution by noting that at maximum plastic deformation, the elastic stress was $\sigma-\sigma_{F}$. When the applied stress is brought back to zero, $-\sigma_{F}$ is substituted for the elastic stress in (4), and the material will be responsive magnetically to this "residual stress." However this residual stress dependence is not the dominant term. (The main phenomenon affecting magnetic behavior is increase in dislocation density during plastic straining.)

By identifying the two contributions to the magnetic changes due to plastic deformation seen in effect as change in the hysteresis width and change in the effective field, we are able to predict 
the magnitudes of changes, like change in coercive field, change in remanent flux density, change in area $\mathrm{W}$ inside the B-H hysteresis loop (called hysteresis loss), and change in maximum differential permeability $(\mathrm{dB} / \mathrm{dH})=\mu_{\max }$. In physical terms, the increase in dislocation density magnetically has the effect of creating a greater density of pinning centers for magnetic domain walls, and the effect also, in the case of dislocation tangles, of creating deeper potential wells to trap the domain walls. This makes the material harder to magnetize and increases its coercivity. This is reflected in an increased domain wall pinning parameter $\mathrm{k}$ in the hysteresis model. In physical terms, the increase in dislocation density magnetically has the effect of creating a greater density of pinning centers for magnetic domain walls, and the effect also, in the case of dislocation tangles, of creating deeper potential wells in which to trap the domain walls. This makes the material harder to magnetize and increases its coercivity. This is reflected in an increased domain wall pinning parameter $\mathrm{k}$ in the hysteresis model.

Since $\mathrm{k}$ is also proportional to the effective field scaling parameter a, it also develops that an increased dislocation density means a smaller effect on the magnetization due to the effective field, since the effective field is divided or scaled by parameter a and this means a reduced slope to the magnetization curve, i.e. reduced differential permeability.

In general, therefore, the magnetic effects of the plastic deformation stem from

1) Dislocation density increases during plastic deformation.

2) A residual plastic strain $e_{r}$ remains when $\sigma$ returns to zero.

The effect of these two attributes is:

1) to linearly increase hysteresis loop width (increase $\mathrm{H}_{\mathrm{c}}$ ) with increased plastic deformation $\mathrm{e}_{\mathrm{r}}$;

2) and to decrease the slope of the loop (decrease $\left.\mu_{\max }\right)$.

This generally agrees with experiment [11].

\section{MODEL RESULTS}

Simulated hysteresis plots are computed using the new formulation.

For this, the Jiles-Atherton parameters are:

- $M_{s}=1.212 \times 10^{6} \mathrm{~A} / \mathrm{m}$,

- $\quad c=6.137 \times 10^{-4}$

- $\alpha=1.270 \times 10^{-4}$,

- for grain size $20 \mu \mathrm{m}$ and dislocation density $1 \times 10^{12} / \mathrm{m}^{2}$ : 


$$
k_{0} / \mu_{0}=34.61 \mathrm{~A} / \mathrm{m} \text { and } a_{0}=56.82 \mathrm{~A} / \mathrm{m} \text {. }
$$

- Constants $G_{1}=0.8100 \times 10^{-6} \mathrm{~m}$ and $G_{2}=3.800 \times 10^{-12} \mathrm{~m}^{2}$

- Poisson's ratio $v=0.333$

Saturation magnetostriction is given by $\lambda_{s}=9.42 \times 10^{-6}$; Young's modulus is $1.78 \times 10^{11} \mathrm{~J} / \mathrm{m}^{3}=$ $178 \mathrm{GPa}$. (from mechanical measurements); hysteresis curves are all taken to $B_{\max }=1.5 \mathrm{~T}$.

The Ludwik constant $C$ for relating the strain-hardening stress $\sigma_{F}$ to the residual plastic strain $e_{r}$ is taken as $C=7.0 \times 10^{20} \mathrm{~J} / \mathrm{m}^{3}$.

Note that the fit to Ludwik's law goes as strain-hardening stress is equal to $\mathrm{C}$ times the additional strain taken to the power n.

$$
\sigma_{F}=C e_{r}^{n}
$$

Computed hysteresis curves are found using Ludwik's equation for $\sigma_{F}$ vs. $e_{r}$ with $n=0.657$ and using (8) for residual stress $\sigma_{F}$. The result of the numerical computations is shown in Fig. 1.

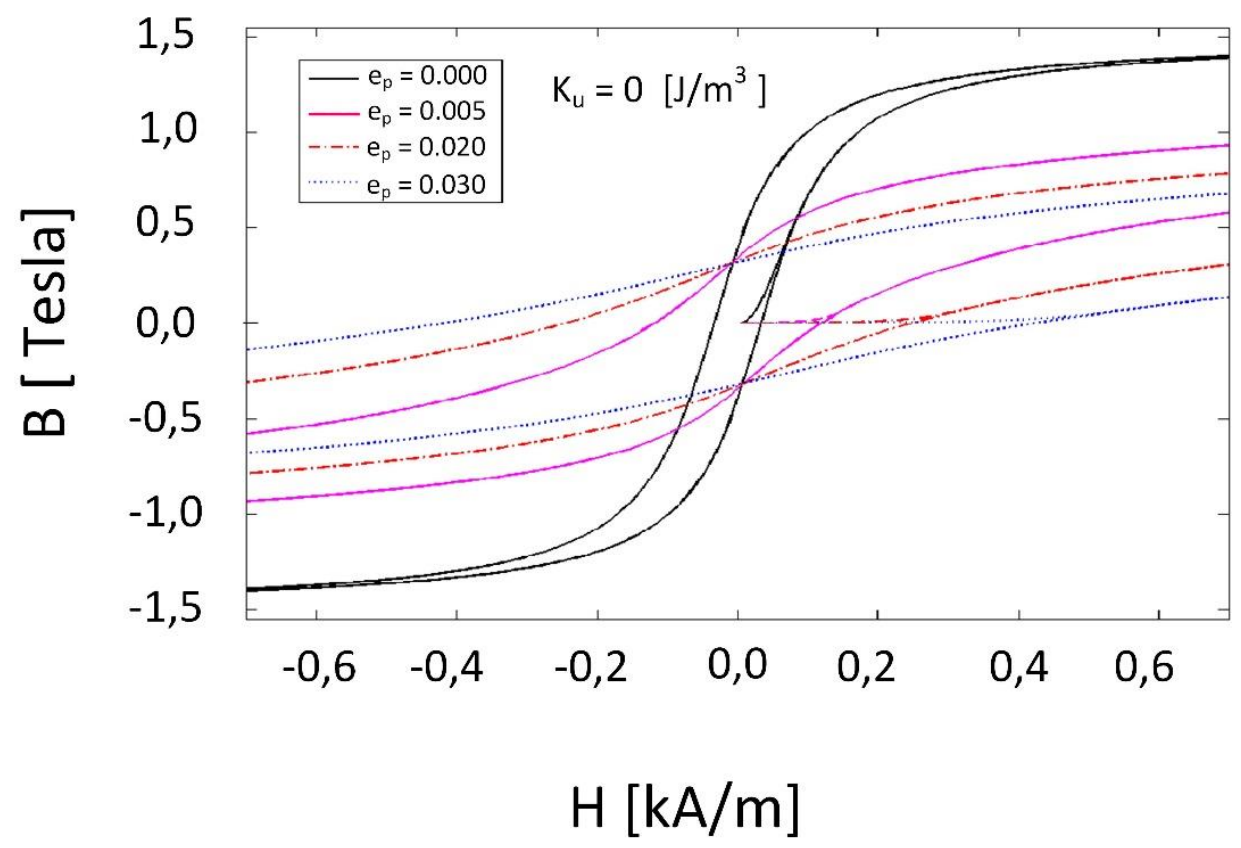

Fig. 1. Hysteresis curves are shown for deformations of $0,0.005,0.02$, and 0.05 . The loops at non-zero deformations are not shown in full, as they are cut off at $\mathrm{H}_{\max }=700 \mathrm{~A} / \mathrm{m}$ 
Note the loops are strongly sheared relative to the zero deformation curve, as seen experimentally.

In Fig. 2 we see the hysteresis loss plotted against the plastic strain.

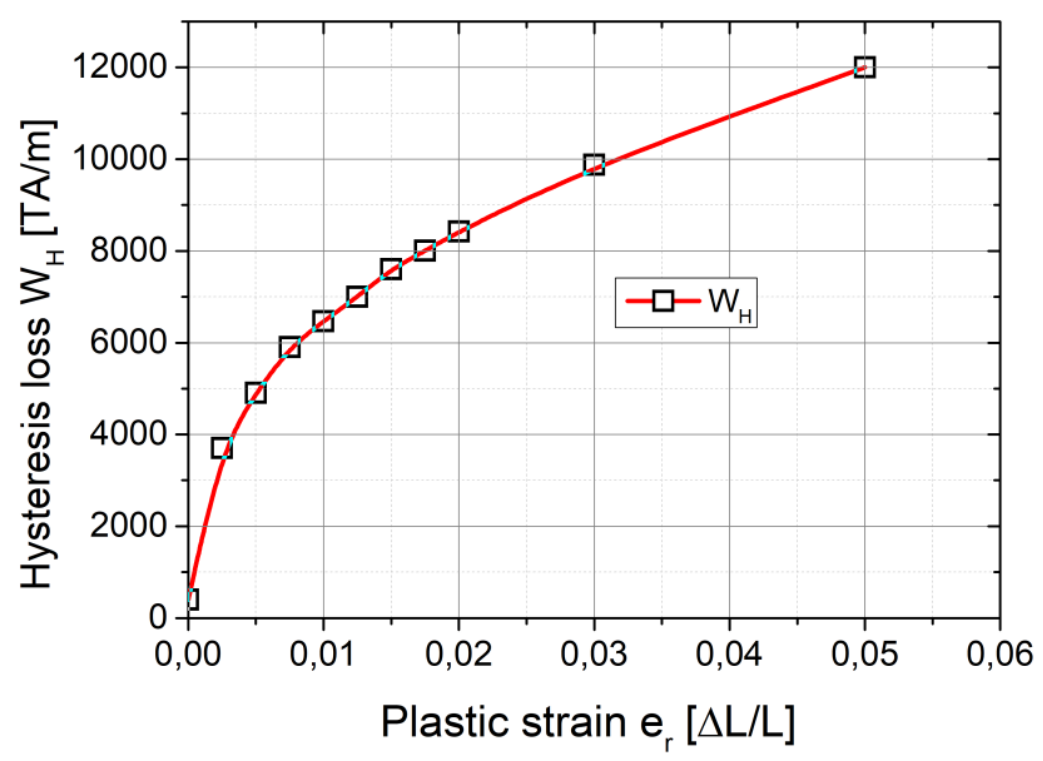

Fig. 2. The curves for hysteresis loss vs. plastic strain are given by the curve above

Fig. 3 shows a replotting of Fig. 2 as a log-log plot. This shows Ludwig Law-like depemdences. The power law dependence of $W_{H}$ on residual plastic strain $e_{r}$, with power law exponent $m=0.270$ is different from mechanical Ludwik exponent $n=0.652$, and is to be compared to measured values of 0.333 and 0.292 . It is almost as if the power law is roughly half that in the mechanical case. 


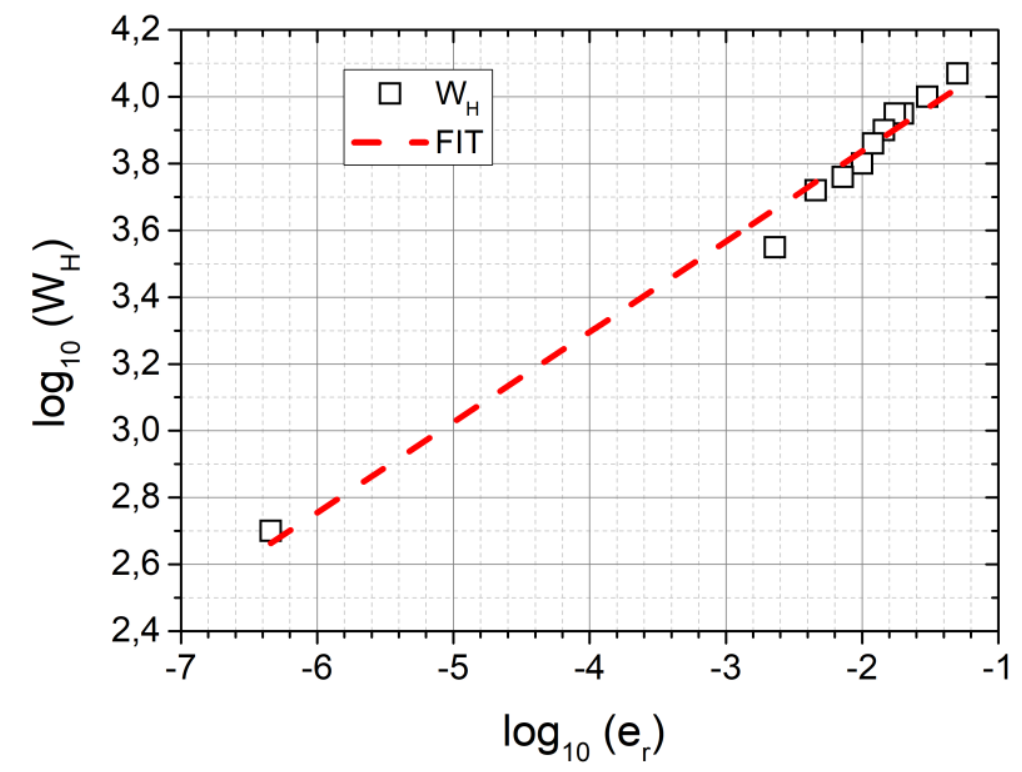

Fig. 3. This curve represents a replotting on a log-log plot to show the Ludwik law-like dependences

\section{SUMMARY}

We have applied a model for tensile deformation effects on magnetic hysteresis. The mechanical part of the model (Ludwik's law) is obtained from a nonlinear extrapolation into the discontinuous yielding region.

The modeling indicates that there should be sharp shearing of the hysteresis loops for the deformed specimens, in contrast to the narrow, sharply step-like loop found for the undeformed specimen.

It is found from the mechanical data that a $\underline{e}_{\underline{\underline{r}}}^{0.657}$ dependence for the strain-hardening stress $\sigma_{\underline{F}}$ exists. It is found also that such a dependence results in the experimentally observed step at low deformation in the $e_{r}$ dependence of $\underline{W}_{\underline{H}}$, which has its own power law dependence of $e_{r}^{0.270}$ (as modeled) and $e_{r}^{0.333}$ or $e_{r}^{0.292}$ (as experimentally found). 


\section{NDE APPLICATION}

One would look to know how to use the modeling for an NDE application e.g., perhaps to know how plastically deformed a material is. The dislocation density might be a measure of that.Let us return to:

To determine cumulative dislocation density $\zeta_{d}$ produced by the plastic deformation, [13,14] we use:

$$
\zeta_{d}^{1 / 2}-\zeta_{d 0}^{1 / 2}=\left|\sigma_{F}\right| /\left(\alpha_{K} G b\right)
$$

where $\zeta_{d 0}$ is the initial dislocation density prior to plastic deformation, $G$ is the specimen shear modulus, $b$ is the appropriate Burgers vector magnitude for the specimen's dislocations, and $\alpha_{K}$ is a constant given as 0.76. "Strain-hardening stress" $\sigma_{F}$ is $\sigma_{F}=\sigma_{f}-\sigma_{y}$. Magnitude $\left|\sigma_{F}\right|$ cannot be negative, and so the dislocation density is monotonically increasing regardless of whether the plastic deformation is tensile or compressive, as must physically be the case. The strainhardening stress can be written in terms of the residual plastic strain, using Ludwik's law.

To get Ludwik's law, we have to do a log-log plot on our sample, thereby obtaining the exponent $\mathrm{n}$ from the slope and the constant $\mathrm{C}$ from the intercept $\log \mathrm{C}$. Once we have Ludwik's Law, we can get the flow stress from the residual deformation. Then, knowing the flow stress, one can obtain the dislocation density.

\section{REFERENCES}

1. Sablik M.J. and Jiles D.C., Coupled magnetoelastic theory of magnetic and magnetostrictive hysteresis, IEEE Trans. Magn. 29 (1993) 2113-2123.

2. Sablik M,J., Rios S., Landgraf F.J.G., et al., Modeling of sharp change in magnetic hysteresis behavior of electrical steel at small plastic deformation, J. Appl. Phys. 97 (2005) 10E518-1 10E518-3.

3. Sablik M.J., Landgraf F.J.G., Modeling microstructural effects on hysteresis loops with the same maximum flux density, IEEE Trans. Magn. 39 (2003) 2528-2530.

4. Sablik M.J., Landgraf F.J.G., Magnabosco R., Fukuhara M., de Campos M.F., Machado R. Missell F.P., Fitting the flow curve of a plastically deformed silicon steel for the prediction of magnetic properties, J. Magn. Magn. Mater. 304 (2006) 155-158.

5. Sablik M.J., Yonamine T., Landgraf F.J.G., Modeling plastic deformation effects on hysteresis loops with the same maximum flux density in steels, IEEE Trans. Mag. 40 (2004) 3219-3226.

6. Szewczyk R., Salach J., Bieńkowski A., Modeling of magnetoelastic materials for force and torque sensors, Solid State Phenom. 144 (2009) 124-129. 
7. Li J., Xu M., Modified Jiles-Atherton-Sablik model for asymmetry in magnetomechanical effect under tensile and compressive stress, J. Appl. Phys. 110(6) (2011) 063918.

8. Zirka S. E., Moroz Y. I., Harrison R. G., Chwastek K., On physical aspects of the Jiles-Atherton hysteresis models, J. Appl. Phys. 112(4) (2012) 043916.

9. Nowicki M., Szewczyk R., Charubin T., Marusenkov A., Nosenko A., Kyrylchuk V., Modeling the hysteresis loop of ultra-high permeability amorphous alloy for space applications, Materials, 11(11) (2018) 2079.

10. Jakubas A., Chwastek K., A Simplified Sablik's Approach to model the effect of compaction pressure on the shape of hysteresis loops in soft magnetic composite cores, Materials, 13(1) 2020, 170.

11. Landgraf F. J. G., Emura M. Losses and permeability improvement by stress relieving fully processed electrical steels with previous small deformations, J. Magn. Magn. Mater. 242 (2002) 152-156.

12. Chady T., Grochowalski J. M. Eddy current transducer with rotating permanent magnets to test planar conducting plates, Sensors, 19(6) (2019) 1408.

13. Kronmuller H., Magnetic techniques for the study of dislocations in ferromagnetic materials, Int. J. Nondestruct. Testing, 3 (1972) 314-321.

14. Astie B., Degauque J. et al., Influence of the dislocation structures on the magnetic and magnetomechanical properties of high-purity iron, IEEE Trans. Magn. 17 (1981) 2929-2931. 